\title{
On sequential selection and a first passage problem for the Poisson process
}

\author{
Alexander Gnedin*
}

\begin{abstract}
This note is motivated by connections between the online and offline problems of selecting a possibly long subsequence from a Poisson-paced sequence of uniform marks under either a monotonicity or a sum constraint. The offline problem with the sum constraint amounts to counting the Poisson arrivals before their total exceeds a certain level. A precise asymptotics for the mean count is obtained by coupling with a nonlinear pure birth process.
\end{abstract}

Keywords: sequential selection; Poisson process; birth process; Borel distribution. MSC2020 subject classifications: 60G55; 60G80; 60C05.

Submitted to ECP on July 21, 2020, final version accepted on January 27, 2021.

\section{Introduction}

When a shuttle carrying a large number of hotel guests arrives at the hotel, the passengers start queuing and pass the exit door at times of a Poisson process. The waiting times spent in the queue are added up as the passengers quit. What is the number $N(t)$ of passengers that exit the shuttle before the accumulated waiting time exceeds $t$ ?

We shall call this the shuttle exit problem. The exit count $N(t)$ is the maximum number of Poisson times with the total not exceeding $t$. The total waiting time and the exit count process are important in many models of applied probability. Our interest stems from the connection to the online version of the longest increasing subsequence problem, which we now describe along with its offline counterpart.

Suppose independent, uniform $[0,1]$ marks arrive sequentially at times of a unit rate Poisson process on $[0, t]$. Plotting each mark against its arrival time yields a Poisson scatter of points in two dimensions. An increasing subsequence is a subset of points making up a chain in the natural partial order. A prophet with complete overview of the data can use an offline algorithm to select the longest increasing subsequence of some length $L^{*}(t)$. The circle of questions about $L^{*}(t)$ is known as the Ulam-Hammersley problem, see Romik's book [17] for a nice exposition. A nonclairvoyant gambler learns the data and makes irrevocable decisions in real time using a nonanticipating online selection policy. Let $L(t)$ be the length of increasing subsequence selected under the online policy that achieves the maximum expected length. As $t \rightarrow \infty$,

$$
\begin{aligned}
\mathbb{E} L^{*}(t) & =2 \sqrt{t}-c_{0} t^{1 / 6}+o\left(t^{1 / 6}\right), \\
\mathbb{E} L(t) & =\sqrt{2 t}-\frac{1}{12} \log t+O(1),
\end{aligned}
$$

*Queen Mary, University of London, E-mail: a.gnedin@qmul.ac.uk 
(where $c_{0}=1.77 \ldots$ is an explicit constant). The limit ratio $2: \sqrt{2}$ serves as a rough measure of advantage of the prophet over the gambler. The asymptotics (1.1) has a long and colourful history, culminating in the work of Baik, Deift and Johansson [3]. The leading term of (1.2) is due to Samuels and Steele [18] who were first to study the online problem, later Bruss and Delbaen [7] identified the logarithmic order of the second term and the full expansion has appeared recently in [13].

Remarkably, the online increasing subsequence problem can be recast as a very different bin-packing problem, where the task is to maximise the expected length of online selected subsequence under the constraint that the sum of selected marks does not exceed 1 (the interpretation of the condition is that the selected items fit in a bin of unit capacity) [6,9]. By analogy with (1.1) and (1.2) it is natural to consider the offline counterpart of $L(t)$ in the bin packing context. Obviously, with full information available, the optimal prophet's policy amounts to the smallest first policy that packs the items in the increasing order of size as long as they fit in the unit bin.

Since the marks sorted into increasing order themselves comprise a homogeneous Poisson process, zooming in the marks scale with factor $t$ and changing the metaphore, it is seen that the number of items packed under the smallest first policy coincides with the exit count $N(t)$ from the shuttle problem we started with.

The first surprise in the online-offline bin packing comparison comes with the fact that the limit prophet-to-gambler ratio is equal to 1 . This follows from the asymptotics $\mathbb{E} N(t) \sim \sqrt{2 t}$, which in turn can be concluded from a benchmark [6, 8, 11, 19] upper bound $\mathbb{E} N(t)<\sqrt{2 t}$, the trivial inequality $L(t) \leq N(t)$ and (1.2). Therefore, to assess the magnitude of prophet's advantage one needs to examine the mean exit count more closely.

In this note we find a formula for $\mathbb{E} N(t)$ in terms of the Borel distribution. Though explicit, the formula seem to require substantial analytic work to extract the desired second term of the asymptotic expansion. We circumvent this by resorting to elementary probabilistic tools, with the core of our approach being the observation that $N(t)$, for each fixed $t$, has the same distribution as the entrance count $M(t)$ appearing in the following dual shuttle entrance problem.

When the shuttle picks up hotel guests at the airport, they enter by the Poisson process. The shuttle departs at the moment when the total waiting time of the driver and all passengers inside the shuttle is $t$. What is the number $M(t)$ of hotel guests in the shuttle by the departure?

We observe that the process $M(t)$ is a nonlinear pure-birth Markov chain which was considered in Kingman and Volkov [15] in the context of gunfight models. Using the identity in distribution we show that

$$
\sqrt{2 t}-\mathbb{E} N(t) \rightarrow \frac{2}{3}
$$

and that the difference is always less than 1 . This contrasts sharply with the second terms in (1.1) and (1.2). For the difference between the prophet and gambler values we have therefore

$$
\mathbb{E} N(t)-\mathbb{E} L(t) \sim \frac{1}{12} \log t
$$

Bruss and Delbaen [7] showed that $L(t)$ is $\mathrm{AN}\left(\sqrt{2 t}, \frac{1}{3} \sqrt{2 t}\right)$ (where AN abbreviates 'asymptotically normal'), see also [13]. We argue that the same is true for $N(t)$. The asymptotic coincidence of variances looks unexpected since the underlying selection policies are very different. We remind that in the increasing subsequence problem the types of the limit distributions of $L^{*}(t)$ and $L(t)$ are different, as the distribution of the maximum offline length $L^{*}(t)$ approaches the Tracy-Widom law from the random matrix theory $[3,17]$. 
This note is a collection of snapshots around (1.3). Various connections are pursued, but to keep the discussion short details of routine proofs are only sketched. Related work on sums of consequitive arrivals for inhomogeneous Poisson process appeared in [2], and on the integrated Poisson process in [20].

The rest of the paper is organised as follows. In the next two sections we describe the selection policies and add insight to what is already known regarding the coupling of online problems and the benchmark upper bound. In sections 4 and 5 we scrutinise the exit-entrance duality. In section 6 we highlight the normal limits. In section 7 we derive a series formula for the mean count and relate it to the Borel distribution. In section 8 we employ the pure birth process to refine the $\sqrt{2 t}$ asymptotics. In section 9 we depoissonise (1.3) to improve upon the well known $[6,8,9,19]$ fixed sample size asymptotics of the smallest first policy. A large deviation bound needed for our arguments is derived in the last section.

Throughout we shall be using the notation

$$
\nu(t):=\mathbb{E} N(t), \quad \sigma^{2}(t):=\operatorname{Var} N(t) .
$$

\section{Coupling of online problems}

We first detail the equivalence between the online increasing subsequence and bin packing problems. They differ by the contraint: in the first problem the selected marks should increase and in the second the total should be at most 1 . The question about explicit coupling was emphasized in Section 5 of Steele [19], where problems with fixed number of arrivals $n$ were discussed.

The distribution of marks in the increasing subsequence problem does not matter (subject to being continuous), while the bin packing problem is not distribution-free. In the special case of uniform $[0,1]$ marks and the bin of unit capacity, the equivalence in terms of the optimal policies has been commonly argued by comparing the dynamic programming equations for the value function [1, 9]. It was also noticed in [9] (p. 455) that the greedy policy which selects every item that fits in the remaining capacity outputs the same length as the policy selecting every consequitive record (a mark bigger than all marks seen so far).

The following construction provides a general coupling in our setting of Poisson arrivals, but it can be readily adjusted to other arrival processes including the discrete time models with fixed or random horizon [1, 11, 18].

Let $\Pi^{\square}$ be a planar Poisson point process with unit rate in the strip $[0, \infty) \times[0,1]$. We endow $\Pi^{\square}$ with the natural filtration of sigma-algebras generated by $\left\{\left.\Pi^{\square}\right|_{[0, t] \times[0,1]}, t \geq 0\right\}$. The generic atom of $\Pi^{\square}$ at location $(\tau, \xi)$ is understood as mark $\xi$ arriving at time $\tau$.

We define an $i$-selection policy to be a nondecreasing, adapted, cádlág jump process $I$ with $I(0)=0$, such that the north-west corners of the graph of $I$ are some atoms $\left(\tau_{k}, \xi_{k}\right)$ of $\Pi^{\square}$ labeled by increase of the time component. This sequence of atoms spanning the graph is an increasing chain in the partial order in two dimensions.

Similarly, we define a $b$-selection policy to be a nondecreasing, adapted, cádlág jump process $B$ with $B(0)=1$ and values in $[0,1]$. We require that each jump be corresponding to an atom $\left(\tau_{k}, \xi_{k}\right)$, so that the jump-time is $\tau_{k}$ and the increment is $\xi_{k}$. Thus the range of $B$ is the sequence of partial sums of $\xi_{1}, \xi_{2}, \ldots$

For a fixed $i$-selection policy $I$, we are going to introduce an invertible random transform $\phi_{I}$ of $[0, \infty) \times[0,1]$, which will map $I$ to a $b$-selection policy with the same path $B=I$. The construction is iterative.

At each step $k$ we shall have $[0, \infty) \times[0,1]$ and its duplicate obtained by a measurepreserving $\beta_{k}$. Start with two identical copies of the strip equipped with Poisson point scatters of $\Pi^{\square}$, and a fixed path of $I$ spanned on some points $\left(\tau_{k}, \xi_{k}\right)$. Let $\beta_{0}$ be the 
identity, and $\xi_{0}=0$. At step $k>0$ only the strip $\beta_{k}\left(\left(\tau_{k}, \infty\right) \times\left[\xi_{k-1}, 1\right]\right)$ undergoes a change which amounts to cutting at height $\xi_{k}-\xi_{k-1}$ by the horizontal line and placing part $\beta_{k}\left(\left(\tau_{k}, \infty\right) \times\left[\xi_{k-1}, \xi_{k}\right]\right)$ atop of $\beta_{k}\left(\left(\tau_{k}, \infty\right) \times\left[\xi_{k}, 1\right]\right)$ with the orientation preserved. The mapping $\beta_{k+1}$ is the composition of $\beta_{k}$ and this surgery. With probability one, each point moves under $\beta_{k}$ 's finitely many times, as the moves may only be associated with $\left(\tau_{k}, \xi_{k}\right)$ 's to the left of this point. Thus we may define $\phi_{I}$ as the composition of all $\beta_{k}$ 's.

Note that $\phi_{I}$ preserves the planar Lebesgue measure and does not alter the time component, so leaving each set $(t, \infty) \times[0,1]$ invariant. Consider the transformed point process $\widehat{\Pi}^{\square}:=\phi_{I}\left(\Pi^{\square}\right)$. By the invariance, $\Pi^{\square}$ and $\widehat{\Pi}^{\square}$ share the same one-dimensional Poisson process of arrival times. Given arrival at time $\tau$, the image of $(\tau, \xi)$ under $\phi_{I}$ is uniformly distributed on $\{\tau\} \times[0,1]$ and is independent of $\left.\Pi^{\square}\right|_{[0, \tau) \times[0,1]}$, hence also independent of $\left.\widehat{\Pi}^{\square}\right|_{[0, \tau) \times[0,1]}$. But this implies that $\widehat{\Pi}^{\square}$ has the same distribution as $\Pi^{\square}$. The transformation $\phi_{I}$ sends the sequence $\left(\tau_{k}, \xi_{k}\right)$ to a sequence $\left(\tau, \xi_{k}-\xi_{k-1}\right)$ (where $\xi_{0}=0$ ), which are now some atoms of $\widehat{\Pi}^{\square}$, and $I$ becomes a $b$-selection policy spanned on the transformed sequence.

The above concepts of selection policy are much more general than the Markovian threshold policies studied in the literature. For the purpose of optimisation, however, it is sufficient to consider the following family of policies. For $\psi:[0, \infty) \rightarrow[0,1]$ thought of as a function controlling the size of acceptance window, and given horizon $t$, an $i$-selection policy is defined recursively by the rule: conditionally on arrival occurring at time $\tau<t$ and given $I(\tau-)=x$ (the last selection so far), the observed mark $\xi$ is selected if and only if

$$
0<\frac{\xi-x}{1-x} \leq \psi((t-\tau)(1-x))
$$

In [13] we called such policies self-similar because the performance from each stage on only depends on the mean number of future arrivals, admissible in the sense that they could be added to the subsequence constructed so far. Thus defined, $I$ is a jump Markov process with transition mechanism determined by $\psi$. The twin $b$-selection policy has the acceptance condition

$$
0<\frac{\xi}{1-x} \leq \psi((t-\tau)(1-x)),
$$

given $B(\tau-)=x$ (the total of selected items so far). The optimal $i$-/b-selection policy is of this form with some control $\psi^{*}$ satisfying

$$
\psi^{*}(z) \sim \sqrt{\frac{2}{z}}-\frac{1}{3 z}, \quad z \rightarrow \infty,
$$

see $[5,7,13]$. In [13] we proved that every policy having $\psi(z) \sim \sqrt{2 / z}$ is within $O(1)$ from the optimality, that is achieves the asymptotics (1.2).

The general Markovian policy differs from (2.1) and (2.2) in that $\psi$ is replaced by arbitrary function of $\tau, t$ and $x$.

\section{The upper bound}

For the rest of this paper the variable $t$ will have the meaning of either the bin capacity (the offline bin-packing contest) or the total waiting time (the shuttle context). We shall use $x$ to denote the time variable of the Poisson process.

Let $\pi_{1}<\pi_{2}<\ldots$ be the points of a unit rate Poisson process $\Pi$ on the positive half-line. The exit count is defined as

$$
N(t):=\max \left\{n: \pi_{1}+\cdots+\pi_{n} \leq t\right\}, \quad t \geq 0,
$$

where $\max \varnothing=0$. 
There is a benchmark upper bound for the mean,

$$
\nu(t)<\sqrt{2 t}, \quad t>0,
$$

that appeared in the Poisson setting in [6] (Example 2.4). Similar inequalities for sums of order statistics from the general distribution are found in [8], also see [19] for extended discussion. We relate (3.1) to an isoperimetric inequality, much in line with the examples from $[5,11]$.

Fix $t$. The set of Poisson points $\pi_{n}$ with $\pi_{1}+\cdots+\pi_{n} \leq t$ is a point subprocess of $\Pi$ with rate function $p_{t}$ satisfying

$$
\nu(t)=\int_{0}^{t} p_{t}(x) \mathrm{d} x, \quad \int_{0}^{t} x p_{t}(x) \mathrm{d} x \leq t, \quad 0 \leq p_{t}(x) \leq 1 .
$$

This suggests a problem from the calculus of variations:

$$
\text { maximise } \int_{0}^{t} q(x) \mathrm{d} x \text {, under the constraints } \int_{0}^{t} x q(x) \mathrm{d} x \leq t, \quad 0 \leq q(x) \leq 1 .
$$

The Lagrangian function becomes

$$
\int_{0}^{t}(\theta-x) q(x) \mathrm{d} x, \quad \text { with } \theta>0
$$

which for given multiplier $\theta$ is maximised by the indicator function $q(x)=1(x \leq \theta)$. Accounting for the constraint, the overall maximum value of the integral is $\sqrt{2 t}$, attained at

$$
\theta^{*}=\sqrt{2 t}, \quad q^{*}(x)=1(x \leq \sqrt{2 t}),
$$

which gives the upper bound (3.1) follows.

Remark Solution $q^{*}$ corresponds to a packing policy that picks all items smaller than the threshold $\sqrt{2 / t}$. The policy violates the (almost sure) sum constraint but meets a weaker mean-value constraint. This policy is online implementable and outputs the number of selections with Poisson $(\sqrt{2 t})$ distribution, so has the variance about three times higher than under the optimal offline (see below) or the optimal online policy $[7,13]$.

\section{The exit-entrance duality}

Consider the shuttle entrance problem. When the $n$th passenger enters the total waiting time of everyone inside the shuttle is

$$
\pi_{1}+2\left(\pi_{2}-\pi_{1}\right)+\cdots+n\left(\pi_{n}-\pi_{n-1}\right)=n \pi_{n}-\left(\pi_{1}+\cdots+\pi_{n-1}\right),
$$

so the entrance count is

$$
M(t):=\max \left\{n: n \pi_{n}-\left(\pi_{1}+\cdots+\pi_{n-1}\right) \leq t\right\} .
$$

We assert that

$$
N(t) \stackrel{d}{=} M(t)
$$

Indeed, since

$$
N(t) \geq n \Leftrightarrow \pi_{1}+\cdots+\pi_{n} \leq t, \quad M(t) \geq n \Leftrightarrow n \pi_{n}-\left(\pi_{1}+\cdots+\pi_{n-1}\right) \leq t,
$$


we need to check that

$$
\pi_{1}+\cdots+\pi_{n} \stackrel{d}{=} n \pi_{n}-\left(\pi_{1}+\cdots+\pi_{n-1}\right) .
$$

Recall that, given $\pi_{n}$, the quotients $\pi_{j} / \pi_{n}, j<n$, are independent from $\pi_{n}$ and jointly distributed like the uniform order statistics. Thus for $u_{1}, u_{2}, \ldots$ iid uniform $[0,1]$ we have

$$
\begin{array}{r}
\pi_{1}+\cdots+\pi_{n}=\pi_{n}\left(1+\frac{\pi_{1}+\cdots+\pi_{n-1}}{\pi_{n}}\right) \stackrel{d}{=} \pi_{n}\left(1+u_{1}+\cdots+u_{n-1}\right) \stackrel{d}{=} \\
\pi_{n}\left(1+\left(1-u_{1}\right)+\cdots+\left(1-u_{n-1}\right)\right) \stackrel{d}{=} \pi_{n}\left(n-\left(u_{1}+\cdots+u_{n-1}\right)\right) \stackrel{d}{=} \\
\pi_{n}\left(n-\frac{\pi_{1}+\ldots \pi_{n-1}}{\pi_{n}}\right)=n \pi_{n}-\left(\pi_{1}+\cdots+\pi_{n-1}\right),
\end{array}
$$

where we used symmetry of the uniform distribution.

It is also instructive to argue in terms of the iid exponentially distributed gaps $\eta_{j}:=\pi_{j}-\pi_{j-1}$ (with the convention $\pi_{0}=0$ ). We have

$$
\begin{aligned}
\pi_{1}+\cdots+\pi_{n} & =n \eta_{1}+(n-1) \eta_{2}+\cdots+\eta_{n} \stackrel{d}{=} \\
\eta_{1}+2 \eta_{2}+\cdots+n \eta_{n} & =\pi_{1}+2\left(\pi_{2}-\pi_{1}\right)+\cdots+n\left(\pi_{n}-\pi_{n-1}\right) .
\end{aligned}
$$

The variables $\zeta_{n}:=\eta_{1}+2 \eta_{2}+\cdots+n \eta_{n}$ are the jump-times of the entrance count process. Thus $(M(t), t \geq 0)$ is a pure-birth process that starts with $M(0)=1$ and moves from state $n$ to state $n+1$ at rate $(n+1)^{-1}$.

The entrance count process has a simple combinatorial interpretation. Think of an urn with one red and some number of white balls. At times of the Poisson process a ball is randomly chosen and replaced to the urn. If the chosen ball is red, a white ball is added to the urn, otherwise the urn composition is not changed. For the process starting with one red ball, $M(t)$ is the number of white balls in the urn at time $t$.

The identity (4.1) only holds for the marginal distributions, and the exit count process $(N(t), t \geq 0)$ is not even Markovian. The driver's waiting time was included in the total waiting time to avoid a shift in the distributional identity. We note in passing that without appealing to (4.1) the upper bound $\mathbb{E} M(t) \leq \sqrt{2 t}$ does not seem at all obvious.

\section{Integrals of the Poisson process}

Let

$$
T(x):=\int_{0}^{x} y \mathrm{~d} \Pi(y)=\sum_{j=1}^{\Pi(x)} \pi_{j}, \quad S(x):=\int_{0}^{x} \Pi(y) \mathrm{d} y=\sum_{j=1}^{\Pi(x)}\left(x-\pi_{j}\right) .
$$

The total waiting time accumulated within the real time $x$ is $T(x)$ in the shuttle exit problem, and $x+S(x)$ in the entrance problem, where $x$ is added to account for driver's waiting time. The integration by parts formula becomes

$$
T(x)=x \Pi(x)-S(x) .
$$

By reversibility of $\Pi$ on $[0, x]$ we have

$$
T(x) \stackrel{d}{=} S(x),
$$

This identity has appeared in [20], where it was concluded analytically from the identity of Laplace transforms. Despite that (5.1) holds for each fixed $x$, the processes are very different: $T$ is a jump process with independent increments, while the paths of $S$ are piecewise linear. 
Plugging for $x$ the Poisson times we obtain a few 'total waiting time paradoxes'. First note the defining recursions

$$
S\left(\pi_{n+1}\right)=S\left(\pi_{n}\right)+n\left(\pi_{n+1}-\pi_{n}\right), \quad T\left(\pi_{n+1}\right)=T\left(\pi_{n}\right)+\pi_{n+1} .
$$

Now, given $\pi_{n+1}$, the variables $T\left(\pi_{n}\right)$ and $S\left(\pi_{n+1}\right)$ have the same distribution, and so unconditionally

$$
T\left(\pi_{n}\right) \stackrel{d}{=} S\left(\pi_{n+1}\right),
$$

in apparent disagreement with (5.1).

Moreover, $S\left(\pi_{n+1}\right) \stackrel{d}{=} S\left(\pi_{n}\right)+\pi_{n}$, which is to be compared with (5.2) and (5.3). This identity is equivalent to

$$
\pi_{n+1}\left(u_{1}+\cdots+u_{n}\right) \stackrel{d}{=} \pi_{n}\left(1+u_{1}+\cdots+u_{n-1}\right),
$$

where the $\pi_{n}$ 's are independent of the iid uniform $u_{j}$ 's. To prove (5.4), one can observe two ways to split $T\left(\pi_{n}\right)$ in independent factors, as $\pi_{n+1}\left(T\left(\pi_{n}\right) / \pi_{n+1}\right)$ and as $\pi_{n}\left(T\left(\pi_{n}\right) / \pi_{n}\right)$, then represent the quotients in brackets in terms of the $u_{j}$ 's. Written explicitly in terms of the Erlang density of $\pi_{n}$ and the Irwin-Hall density of the sum of uniforms, (5.4) looks rather involved. See [12] for other exponential-uniform identities derived from the planar Poisson process.

Next, we can represent the exit and entrance counts by means of a time-changed Poisson process. Let $X(t)=\min \{x: T(x)>t\}$ be the right-continuous inverse of $T$, with $X(0)=\pi_{1}$. We can take here min rather than infinum since $T$ jumps at the discrete set of Poisson points. We have then

$$
N(t)=\Pi(X(t))-1 .
$$

The process $S(x)+x$ is strictly increasing, so there is a well defined inverse process $\tau$ with $S(\tau(t))+\tau(t)=t$ and

$$
\frac{\mathrm{d} \tau}{\mathrm{d} t}=\frac{1}{\Pi(\tau(t))+1} .
$$

The entrance counting process satisfies

$$
M(t)=\Pi(\tau(t)) .
$$

The last two formulas give yet another proof that the entrance count is a pure-birth process with the jump rate $(n+1)^{-1}$ at state $M(t)=n$.

\section{Normal limits}

Note that $T$ has independent increments. An application of Campbell's formula yields the moments

$$
\mathbb{E} T(x)=\frac{1}{2} x^{2}, \quad \operatorname{Var} T(x)=\frac{1}{3} x^{3},
$$

and, more generally, the moment generating function

$$
\mathbb{E} e^{z T(x)}=\exp \left(\frac{e^{z x}-z x-1}{z}\right) .
$$

Inverting this, Suyono and van der Weide [20] found the density of $T(x)$ in terms of modified Bessel functions (note that $T(x)$ has mass $e^{-x}$ at zero).

Routine applications of the law of large numbers and the central limit theorem show that for $x \rightarrow \infty$

$$
T(x), S(x) \sim \frac{1}{2} x^{2} \text { a.s., and are } \mathrm{AN}\left(\frac{1}{2} x^{2}, \frac{1}{3} x^{3}\right) .
$$


These relations are readily translated in terms of the inverses, giving for $t \rightarrow \infty$

$$
X(t), \tau(t) \sim \sqrt{2 t} \text { a.s., and are } \mathrm{AN}\left(\sqrt{2 t}, \frac{1}{3} \sqrt{2 t}\right) .
$$

For instance, the normal limit for $X(t)$ follows from (6.1) and the identity $\mathbb{P}(T(x)<t)=$ $\mathbb{P}(X(t)>x)$ applied with

$$
t=\frac{1}{2} x^{2}+z \sqrt{\frac{1}{3} x^{3}}, \quad x=\sqrt{2 t}-z \frac{2^{1 / 4}}{3^{1 / 2}} t^{1 / 4}+O\left(t^{-1 / 4}\right) .
$$

The asymptotics (6.1) combined with (5.5), (5.6) and a large deviation bound for $\Pi(x)$ can be pursued to obtain for $t \rightarrow \infty$

$$
N(t), M(t) \sim \sqrt{2 t} \text { a.s., and are } \mathrm{AN}\left(\sqrt{2 t}, \frac{1}{3} \sqrt{2 t}\right) .
$$

A more direct way relies on the representation

$$
M(t)=\max \left\{n: \zeta_{n} \leq t\right\},
$$

with $\zeta_{n}=\eta_{1}+2 \eta_{2}+\cdots+n \eta_{n}$ and iid, unit rate exponential $\eta_{j}$ 's. To arrive at (6.3) we use that

$$
\zeta_{n} \sim a_{n} \text { a.s., } \quad \text { and is } \operatorname{AN}\left(a_{n}, b_{n}^{2}\right),
$$

where

$$
a_{n}:=\frac{1}{2} n(n+1), \quad b_{n}:=\frac{1}{6} n(n+1)(2 n+1)
$$

are the mean and the variance of $\zeta_{n}$, asymptotic to $n^{2} / 2$ and $n^{3} / 3$, respectively.

The normal limit (6.3) suggests asymptotics of the variance

$$
\sigma^{2}(t) \sim \frac{1}{3} \sqrt{2 t} .
$$

For the time being we shall take the formula for granted, deferring its justification, by checking a uniform integrability condition, to the last section of this paper.

\section{Connection to the Borel distribution}

Recall that $\zeta_{n}=\sum_{j=1}^{n} j \eta_{j}$ (with the $\eta_{j}$ 's being iid exponential) has the same distribution as the entrance total waiting time $S\left(\pi_{n}\right)+\pi_{n}$.

The Laplace transform of $\zeta_{n}$ is

$$
\mathbb{E} \exp \left(z \zeta_{n}\right)=\prod_{j=1}^{n} \frac{1}{1-j z} .
$$

Inverting this yields a formula for the distribution function

$$
\mathbb{P}(M(t) \geq n)=\mathbb{P}\left(\zeta_{n} \leq t\right)=\frac{1}{n !} \sum_{j=1}^{n}\left(\begin{array}{l}
n \\
j
\end{array}\right)(-1)^{n-j} j^{n}\left(1-e^{-t / j}\right) .
$$

See [21] for an asymptotic expansion for large $t$.

For the mean of $N(t)$, with a small series work, we obtain an exact formula

$$
\nu(t)=\sum_{n=1}^{\infty} \mathbb{P}\left(\zeta_{n} \leq t\right)=\sum_{j=1}^{\infty} e^{-j} \frac{j^{j}}{j !}\left(1-e^{-t / j}\right) .
$$

Obviously, (7.1) can be viewed as a mean

$$
\nu(t)=\mathbb{E}\left(Z\left(1-e^{-t / Z}\right)\right)
$$


over the Borel(1) distribution

$$
\mathbb{P}(Z=j)=e^{-j} \frac{j^{j-1}}{j !}, j=1,2, \cdots,
$$

which has familiar interpretations as

(i) the total population in the critical Galton-Watson process with the Poisson(1) offspring,

(ii) the hitting time for a linear boundary $Z \stackrel{d}{=} \min \{n: \Pi(n)=n-1\}$.

However, we could not find a construction explaining why the Borel distribution appears in our context.

\section{Bounds on the mean and the limit constant}

The transition probability of the entrance count process $M$ is

$$
\mathbb{P}(M(t+d t)-M(t)=1 \mid M(t))=\frac{d t}{M(t)+1},
$$

which upon taking the expectation becomes

$$
\nu^{\prime}(t)=\mathbb{E}\left(\frac{1}{M(t)+1}\right) .
$$

Applying Jensen's inequality we arrive at a differential inequality

$$
\nu^{\prime}(t)>\frac{1}{\nu(t)+1},
$$

which is readily solved by separating variables as $\nu(t)>\sqrt{2 t+1}-1, \quad t>0$.

So together with (3.1) we have fairly tight bounds

$$
\sqrt{2 t+1}-1<\nu(t)<\sqrt{2 t}, \quad t>0,
$$

where the gap stays below 1 for all $t$. The bounds (8.2) clearly suggest that the gap converges to a constant.

Next, we aim at finding the limiting constant suggested by (8.2). The random variable

$$
M(t)+1=\min \left\{n: \zeta_{n}>t\right\}
$$

is a stopping time. Doob's optional sampling theorem applied to the martingale $\zeta_{n}-a_{n}$ yields a Wald-type identity

$$
\mathbb{E}\left[\zeta_{M(t)+1}-\frac{1}{2}(M(t)+1)(M(t)+2)\right]=0 .
$$

On the other hand, conditionally on $M(t)=n$ the distribution of $\zeta_{M(t)+1}-t$ is exponential with rate $(n+1)^{-1}$, so unconditionally we can write the identity in distribution

$$
\zeta_{M(t)+1} \stackrel{d}{=} t+(M(t)+1) \eta,
$$

where $\eta$ is a unit exponential random variable, independent of $M(t)$. Thus

$$
\mathbb{E} \zeta_{M(t)+1}=t+\mathbb{E} M(t)+1,
$$

which together with (8.3) give

$$
\mathbb{E} M^{2}(t)=2 t-\mathbb{E} M(t) .
$$


Alternatively, (8.5) can be derived from the $k=2$ instance of the formula

$$
\left(\mathbb{E} M^{k}(t)\right)^{\prime}=\mathbb{E}\left(\frac{(M(t)+1)^{k}-M^{k}(t)}{M(t)+1}\right)
$$

generalising (8.1). Expanding the right-hand side, it is seen that all moments $\mathbb{E} M^{k}(t)$ can be expressed, recursively, via the first moment $\nu(s), s \leq t$.

Formula (8.5) allows us to express the variance through the mean as

$$
\sigma^{2}(t)=2 t-\nu^{2}(t)-\nu(t)
$$

Substituting the lower bound (8.2) in (8.6) yields the bound $\sigma^{2}(t)<\sqrt{2 t+1}-1$, which for large $t$ is too far from (yet to be justified) (6.4). But working the other way round we substitute (6.4) with indefinite smaller order remainder in (8.6), and work out the quadratic equation to extract the value of the sought limit constant:

$$
\sqrt{2 t}-\nu(t) \rightarrow \frac{2}{3}, \quad \text { as } t \rightarrow \infty .
$$

This result contrasts expansions (1.1) and (1.2) but brings to mind some analogy with the expansion of the classic renewal function in the setting with uniform interarrival times [4] (Ch. 11, Example 8). Numerical calculations with (7.1) suggest that the limit is approached monotonically from below. By analogy with [7, 13], one can conjecture that the next term of the asymptotic expansion of the mean is of the order of $t^{-1 / 2}$, see also [10] for a similar situation.

Remark For random variable $Z$ with Borel(1) distribution, from (8.7) follows the asymptoic expansion for the truncated mean

$$
\mathbb{E}[Z 1(Z \leq z)]=\sqrt{\frac{2 z}{\pi}}-\frac{2}{3}+o(1), \quad z \rightarrow \infty .
$$

This is obtained by writing (7.2) as a Laplace transform

$$
\nu(t)=t \int_{0}^{\infty} e^{-t y} \mathbb{E}\left[Z 1\left(Z \leq y^{-1}\right)\right] \mathrm{d} y
$$

and applying the Tauberian theorem.

Remark Formulas (8.1) and (8.5) generalise. Suppose for the time being that $M$ is a pure-birth Markov process $M$ with $M(0)=0$, and transition rates $\beta_{n}, n \geq 0$, meeting the regularity condition $\sum_{n=0}^{\infty} \frac{1}{\beta_{n}}=\infty$. The mean population size $\nu(t):=\mathbb{E} M(t)$ and the second moment satisfy then $\nu^{\prime}(t)=\mathbb{E} \beta_{M(t)}$ and $\left(\mathbb{E} M^{2}(t)\right)^{\prime}=\mathbb{E}\left[(2 M(t)+1) \beta_{M(t)}\right]$. The identity (8.5) extends as

$$
t=\mathbb{E}\left(\sum_{n=0}^{M(t)-1} \frac{1}{\beta_{n}}\right) .
$$

\section{The smallest first policy for fixed sample size}

We turn to the smallest first policy in the bin packing problem with unit capacity and fixed sample size $n$. Let $0<u_{n 1}<\cdots<u_{n n}<1$ be the uniform $[0,1]$ order statistics, and let

$$
K_{n}=\max \left\{k: u_{n 1}+\cdots+u_{n k} \leq 1\right\} .
$$


be the smallest first count, $\varkappa_{n}:=\mathbb{E} K_{n}$. An explicit formula for $\varkappa_{n}$ exists (see [9], Theorem 7), but is not particularly user-friendly, as it involves an alternating double sum. It is well known that $\varkappa_{n} \sim \sqrt{2 n}$ and that $\sqrt{2 n}$ is also an upper bound $[8,9,11,18,19]$.

We assert now a much more precise result:

$$
\varkappa_{n}=\sqrt{2 n}-\frac{2}{3}+o(1), \quad n \rightarrow \infty .
$$

To show this, we first resort to the setting of the Poisson process on $[0,1]$ with rate $t$. The exit count $N(t)$ translates as the maximal number of Poisson points whose total is at most 1 . Given that the number of all Poisson points is $n$, this count coincides with $K_{n}$, therefore we have the poissonisation relation

$$
\nu(t)=\sum_{n=1}^{\infty} \varkappa_{n} e^{-t} \frac{t^{n}}{n !} .
$$

To depoissonise, we check conditions of Theorem 1 from [14]. To that end, we extend the function $\nu(t)$ given by (7.1) to the complex argument $t \in \mathbb{C}$, thus obtaining an entire function as the series converges everywhere. Some analytic work with the aid of the Stirling formula shows that $|\nu(t)|<c_{1}|t|^{1 / 2}$ in the sector $|\arg t|<\pi / 4$. Outside the sector, we have an estimate

$$
\left|e^{t} \nu(t)\right|<c_{2}|t|^{1 / 2} \exp (|t| / \sqrt{2}) .
$$

This follows by observing that the maximum of $\left|e^{t}\left(1-e^{-t / j}\right)\right|$ for given $|t|$ is achieved at the boundary $|\arg t|=\pi / 4$, and then by approximating the sum (7.1) by an integral. The cited theorem gives the poissonisation error

$$
\left|\nu(t)-\varkappa_{\lfloor t\rfloor}\right|=O\left(t^{-1 / 2}\right), \quad t \rightarrow \infty
$$

hence (8.7) implies (9.1).

The general bin size More generally, suppose the bin has capacity $C>0$. Consider the smallest first policy applied to the Poisson process on $[0,1]$ with rate $t$ and, in parallel, to $n$ items sampled from the uniform $[0,1]$ distribution. Extending our notation from the case $C=1$, let $N_{C}(t)$ and $K_{C, n}$ be the counts of items packed, and let $\nu_{C}(t), \varkappa_{C, n}$ be their means, respectively.

Generalising the $C=1$ result, we argue that

$$
\nu_{C}(t)=\sqrt{2 C t}-\frac{2}{3}+o(1) \text { and }\left|\nu_{C}(t)-\varkappa_{C,\lfloor t\rfloor}\right|=O\left(t^{-1 / 2}\right) .
$$

For $C \leq 1$, this is straightforward, as $\nu_{C}(t)=\nu(C t)$ and we readily conclude (9.2) from the $C=1$ case.

For $C>1$ we need to be more careful since the item sizes are constrained by 1 and not by the bin capacity $C$. Assessing the mean in terms of the unit Poisson process on $[0, C t]$ we have

$$
\begin{array}{r}
\nu_{C}(t)=\sum_{n=1}^{\infty} \mathbb{P}\left(N_{C}(t) \geq n\right)=\sum_{n=1}^{\infty}\left\{\mathbb{P}\left(T\left(\pi_{n}\right) \leq C t\right)-\mathbb{P}\left(T\left(\pi_{n}\right) \leq C t, \pi_{n}>t\right)\right\}= \\
\nu(C t)-\sum_{n=1}^{\infty} \mathbb{P}\left(T\left(\pi_{n-1}\right) \leq(C-1) t, \pi_{n}>t\right) .
\end{array}
$$

Recalling (5.3), we get the identity $\left(T\left(\pi_{n-1}\right), \pi_{n}\right) \stackrel{d}{=}\left(S\left(\pi_{n}\right), \pi_{n}\right)$, hence the last sum becomes

$\sum_{n=1}^{\infty} \mathbb{P}\left(S\left(\pi_{n}\right) \leq(C-1) t, \pi_{n}>t\right) \leq \sum_{n=1}^{\lfloor t / 2\rfloor} \mathbb{P}\left(\pi_{n}>t\right)+\sum_{n=\lfloor t / 2\rfloor+1}^{\infty} \mathbb{P}\left(S\left(\pi_{n}\right) \leq(C-1) t\right)=: \Sigma_{1}+\Sigma_{2}$. 
As $t \rightarrow \infty$, a large deviation estimate for the Poisson process shows that $\Sigma_{1}$ approaches 0 exponentially fast, and the same is shown for $\Sigma_{2}$ using $S\left(\pi_{n}\right)=\zeta_{n}$ and the large deviation estimate (10.1) in the next section.

\section{A large deviation bound}

To justify asymptotics of the variance (6.4) it remains to verify that the family

$$
\frac{M(t)-\sqrt{2 t}}{\left(\frac{1}{3} \sqrt{2 t}\right)^{1 / 2}}, \quad t>0,
$$

is uniformly integrable.

We consider first

$$
\zeta_{n}-a_{n}=\sum_{j=1}^{n} j\left(\eta_{j}-1\right) .
$$

For $\eta$ with the unit exponential distribution, the central moments are estimated as

$$
\mathbb{E}(\eta-1)^{m}=m ! \sum_{j=1}^{m} \frac{(-1)^{j}}{j !}<\frac{m !}{e}+1 .
$$

Using this it is easy to check that

$$
\mathbb{E}[k(\eta-1)]^{m} \leq \frac{k^{2}}{2} n^{m-2} m !, \quad 1 \leq k \leq n, m \geq 2,
$$

which verifies the condition for large deviation bounds from [16] (Chapter 3, Theorem 17). Hence we obtain

$$
\sup _{z \geq 0} \mathbb{P}\left(\left|\zeta_{n}-a_{n}\right|>b_{n} z\right)<3 e^{-z / 4},
$$

where both constants are not sharp. Inverting the latter we arrive at similar bound

$$
\mathbb{P}\left(|M(t)-\sqrt{2 t}|>z\left(\frac{1}{3} \sqrt{2 t}\right)^{1 / 2}\right)<c e^{-z^{2} / 4},
$$

which implies the desired uniform integrability.

\section{References}

[1] Arlotto, A., Mossel, E. and Steele, J. M.: Quickest online selection of an increasing subsequence of specified size. Random Structures Algorithms 49, (2016), 235-252. MR-3536538

[2] Arratia, R., Kochman, F. and Zabell, S.: Large deviation asymptotics for a random variable with Lévy measure supported by $[0,1]$. (2016), arXiv:1606.03524.

[3] Baik, J., Deift, K. and Johansson, K. (1999). On the distribution of the length of the longest increasing subsequence of random permutations. J. Amer. Math. Soc. 12, (1999), 1119-1178. MR-1682248

[4] Feller, W.: An introduction to probability theory and its applications. vol 2., Wiley, 1966. xviii+636 pp. MR-0210154

[5] Baryshnikov, Y. and Gnedin, A.: Sequential selection of an increasing sequence from a multidimensional random sample. Ann. Appl. Probab. 10, (2000), 258-267. MR-1765211

[6] Boshuizen, F.A. and Kertz, P.R.: Smallest-fit selection of random sizes under a sum constraint: weak convergence and moment comparisons. Adv. Appl. Prob. 31, (1999), 178-198. MR1699667

[7] Bruss, F. T. and Delbaen, F.: A central limit theorem for the optimal selection process for monotone subsequences of maximum expected length. Stoch. Proc. Appl. 114, (2004), 287-311. MR-2101246 
[8] Bruss, F.T. and Robertson, J.: Wald's lemma for sums of order statistics of I.I.D. random variables. Adv. Appl. Prob. 23, (1991), 612-623. MR-1122878

[9] Coffman, E.G, Flatto, L. and Weber, R.R.: Optimal selection of stochastic intervals under a sum constraint. Adv. Appl. Prob. 19, (1987), 454-473. MR-0889945

[10] Coffman, E.G., Flajolet, P., Flatto, L. and Hofri, M.: The maximum of a random walk and its application to rectangle packing. Probab. Engrg. Inform. Sci. 12, (2001), 373-386. MR-1631315

[11] Gnedin, A.: Sequential selection of an increasing subsequence from a sample of random size, J. Appl. Probab. 36, (1999), 1074-1085. MR-1742151

[12] Gnedin, A. and Marynych, A.: Exponential-uniform identities related to records. Electron. Commun. Probab. 17, (2012), paper no. 26. MR-2955491

[13] Gnedin, A. and Seksenbayev, A.: Asymptotics and renewal approximation in the online selection of an increasing subsequence, to appear in Bernoulli (2021).

[14] Jacquet, P. and Szpankowski, W.: Analytical de-Poissonization and its applications. Theoret. Comput. Sci. 201, (1998), no. 1-2, 1-62. MR-1625392

[15] Kingman, J.F.C. and Volkov, S.E.: Solution to the OK Corral model via decoupling of Friedman's urn. J. Theoret. Probab. 16, (2003), 1-10, MR-1956831; Corrected in J. Theor. Probab. 32, 1614-1615. MR-3979683

[16] Petrov, V.: Sums of Independent Random Variables. Translated from the Russian by A. A. Brown. Ergebnisse der Mathematik und ihrer Grenzgebiete, Band 82. Springer, 1975. x+346 pp. MR-0388499

[17] Romik, D.: The Surprising Mathematics of Longest Increasing Subsequences. Cambridge University Press, 2015. MR-3468738

[18] Samuels, S.M. and Steele, J.M.: Optimal sequential selection of a monotone sequence from a random sample. Ann Probab. 9, (1981), 937-947. MR-0632967

[19] Steele, J.M. (2016) The Bruss-Robertson inequality: elaborations, extensions, and applications. Mathematica Applicanda 44, 3-16. MR-3557088

[20] Suyono and van der Weide, J.A.M.: Integrated renewal process. J. Indones. Math. Soc. (MIHMI) 13, (2007), 149-159. MR-2396753

[21] Van Leeuwaarden, J. S. H. and Temme, N. M.: A uniform asymptotic expansion for weighted sums of exponentials. Statist. Probab. Lett. 81, (2011), 1571-1579. MR-2832914

Acknowledgments. The author is grateful Alex Marynych for a number of illuminating discussions. Thanks go to an anonymous referee for a pointer to the literature and contructive comments that lead to improvement of the presentattion. 


\section{Electronic Journal of Probability Electronic Communications in Probability}

\section{Advantages of publishing in EJP-ECP}

- Very high standards

- Free for authors, free for readers

- Quick publication (no backlog)

- Secure publication $\left(\mathrm{LOCKSS}^{1}\right)$

- Easy interface (EJMS²)

\section{Economical model of EJP-ECP}

- Non profit, sponsored by $\mathrm{IMS}^{3}, \mathrm{BS}^{4}$, ProjectEuclid ${ }^{5}$

- Purely electronic

\section{Help keep the journal free and vigorous}

- Donate to the IMS open access fund ${ }^{6}$ (click here to donate!)

- Submit your best articles to EJP-ECP

- Choose EJP-ECP over for-profit journals

\footnotetext{
${ }^{1}$ LOCKSS: Lots of Copies Keep Stuff Safe http://www. lockss.org/

${ }^{2}$ EJMS: Electronic Journal Management System http://www.vtex.lt/en/ejms.html

${ }^{3}$ IMS: Institute of Mathematical Statistics http://www.imstat.org/

${ }^{4}$ BS: Bernoulli Society http://www. bernoulli-society.org/

${ }^{5}$ Project Euclid: https://projecteuclid.org/

${ }^{6}$ IMS Open Access Fund: http://www.imstat.org/publications/open.htm
} 